\title{
Backscatter Mapping, Faunal \\ Sampling and Benthic Habitat in the Long Island Sound Cable Fund Seafloor Habitat Mapping Project
}

\author{
Roger D Flood and Robert M Cerrato \\ School of Marine and Atmospheric Sciences \\ Stony Brook University \\ Stony Brook, NY 11794-5000
}

honorable mention: Alison Flannigan, Nichole Maher, Lee Holt, Juliet Kinney, Vicki Ferrini, Michael White 


\section{Water Depth}

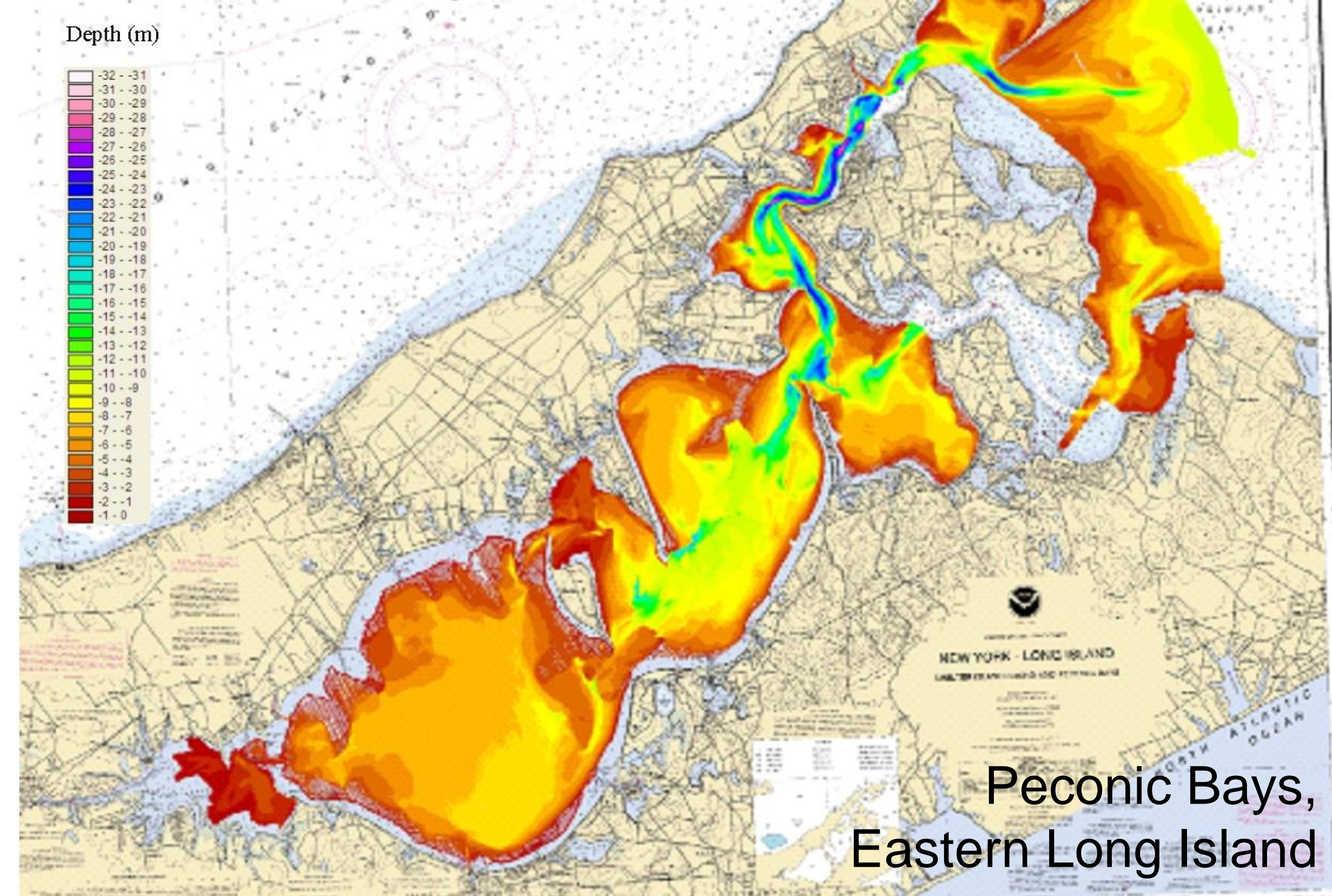


Hillshade (Sun Illuminated Bathy)

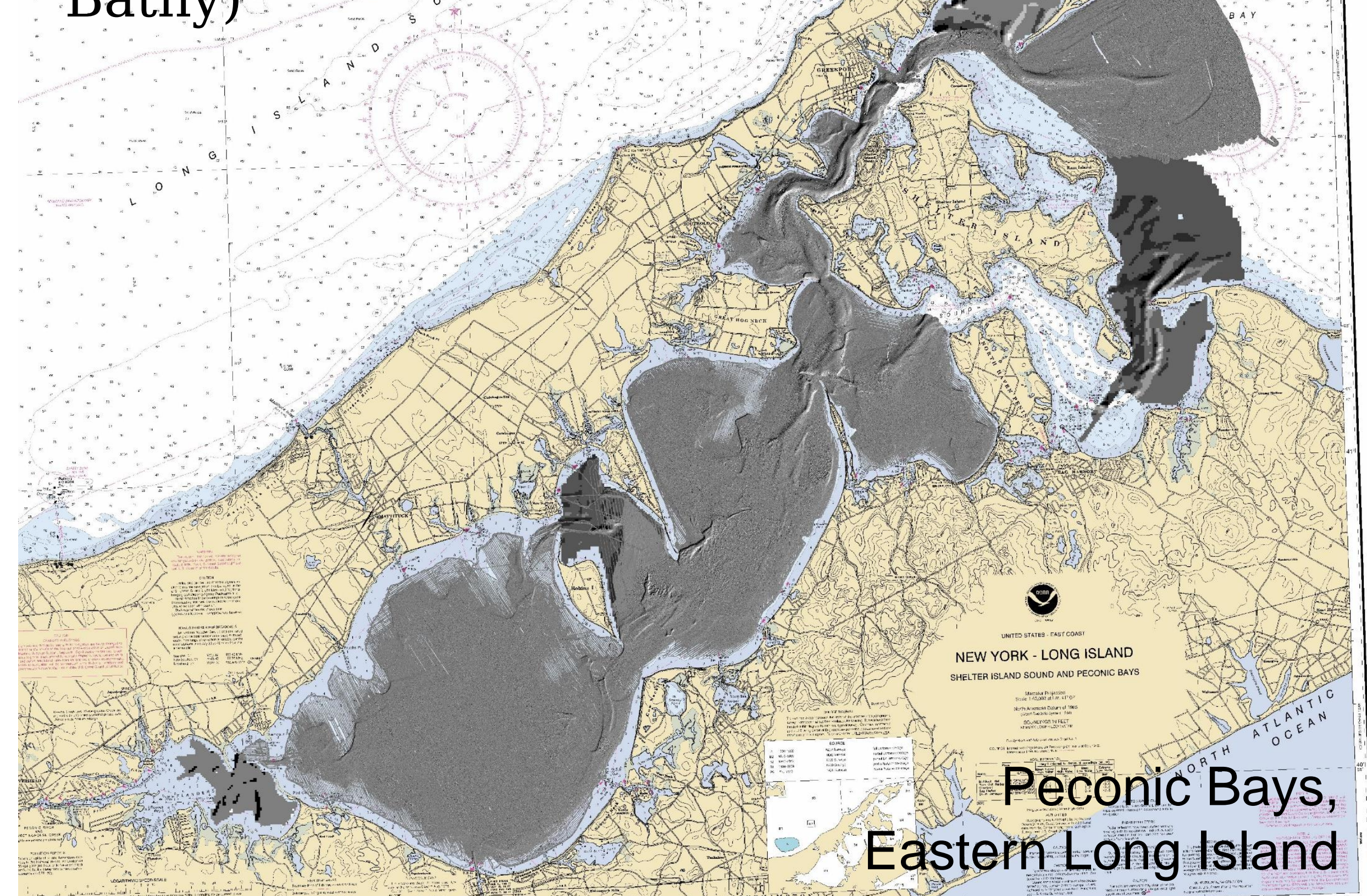


Backscatter

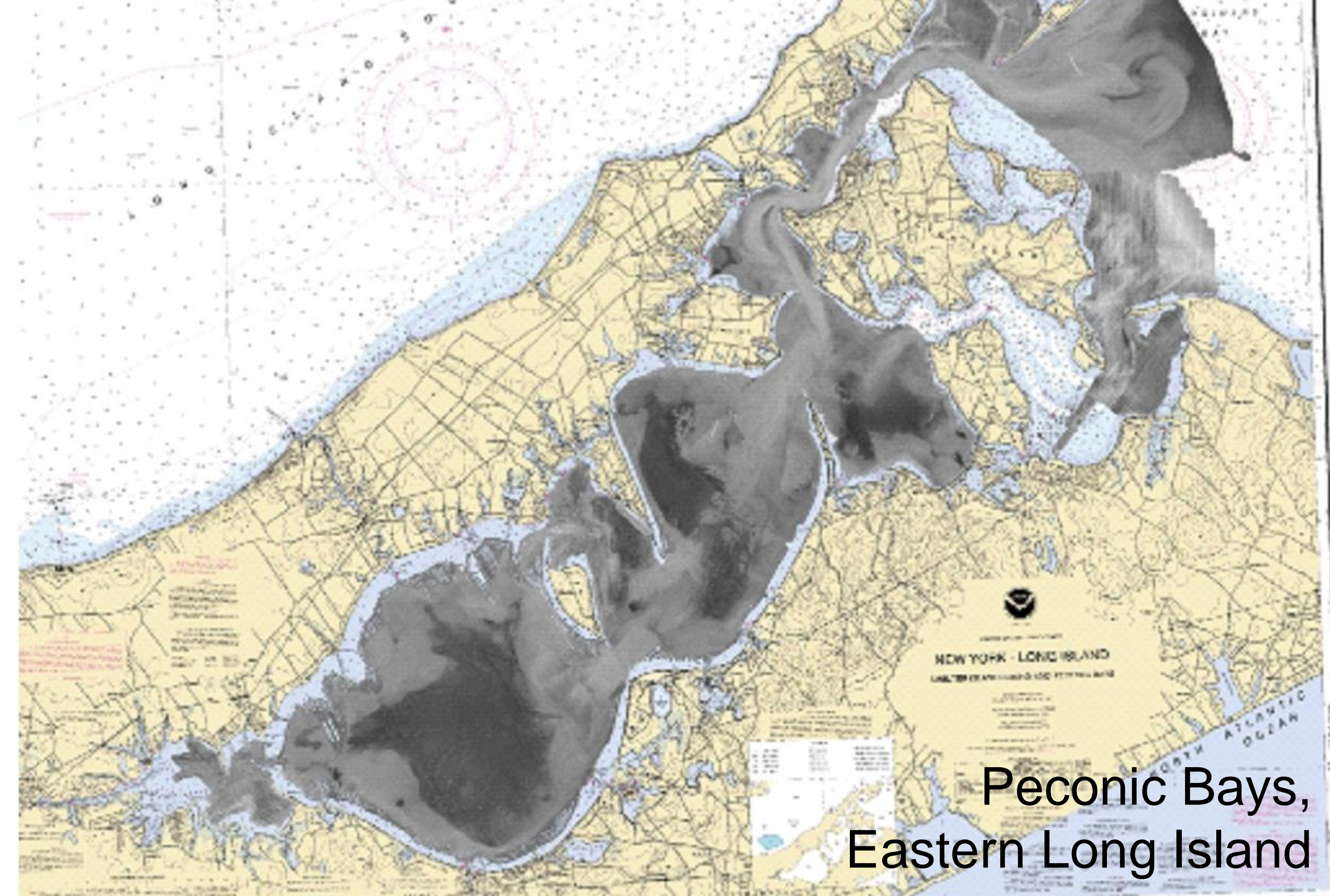



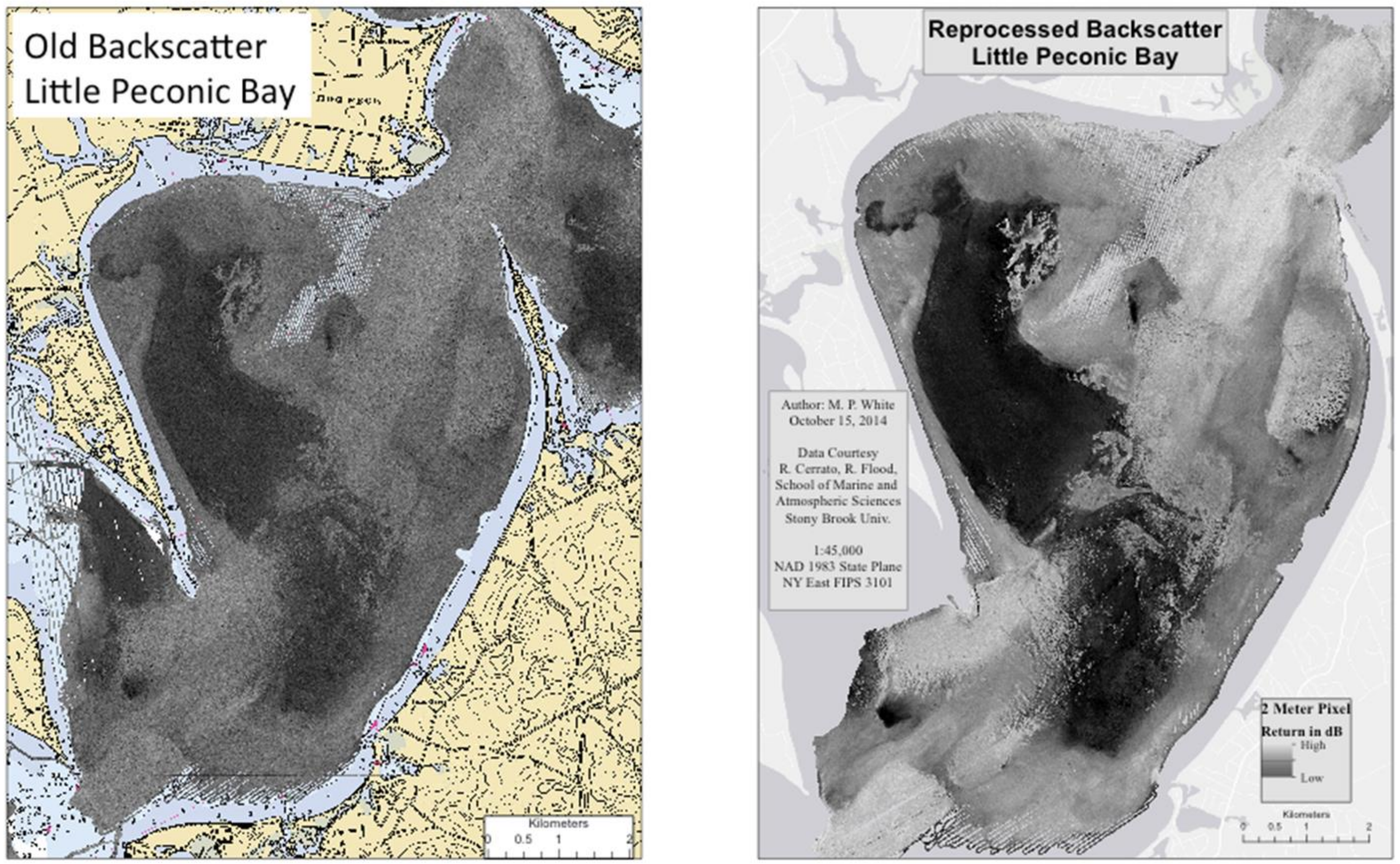
Effects of Benthos on Sediment Acoustic Properties

- Create variable transition layer between water and sea bed (e.g., fecal pellets, epiflora, epifauna).

- Alter bulk density value and profile in near-surface sediment (e.g., feeding voids, tube clusters).

- Alter surface roughness and create patchiness (e.g., shell, fecal pellets, tracks \& trails, mounds).

- Increase volume scattering (e.g., shell and large-particles at depth).

- Alter sediment transport effects and patterns due to sediment binding.
Acoustic Properties that affect Backscatter (many of these effects are frequency dependent)

- Surface grain size

- Surface roughness

- Volume scattering

- Density, porosity and microstructure

- Sound velocity value and structure

Can we use acoustic backscatter as another tool for assessing the distribution of benthic communities (groups of organisms that live together). However, while different benthic communities may result in different acoustic backscatter patterns, we know that many other factors affect acoustic backscatter. 


\section{Benthic Habitat Mapping Research Goals}

- Characterize underwater lands using sonar and other mapping techniques, AND SAMPLING

- Describe natural processes and history of underwater lands

- Understand substrate factors important in distribution of benthos

- Quantify type and distribution of benthos and identify biotopes (distinctive assemblages of benthic species)

- Improve predictive capability of benthic habitats and biotopes

- Provide information to managers (habitat ranges, sensitivity to loss/degradation, baseline for future change, information about rare species) 
North Shore Harbors: Port Jefferson Harbor, Huntington Bay \& Cold Spring - Oyster Bay

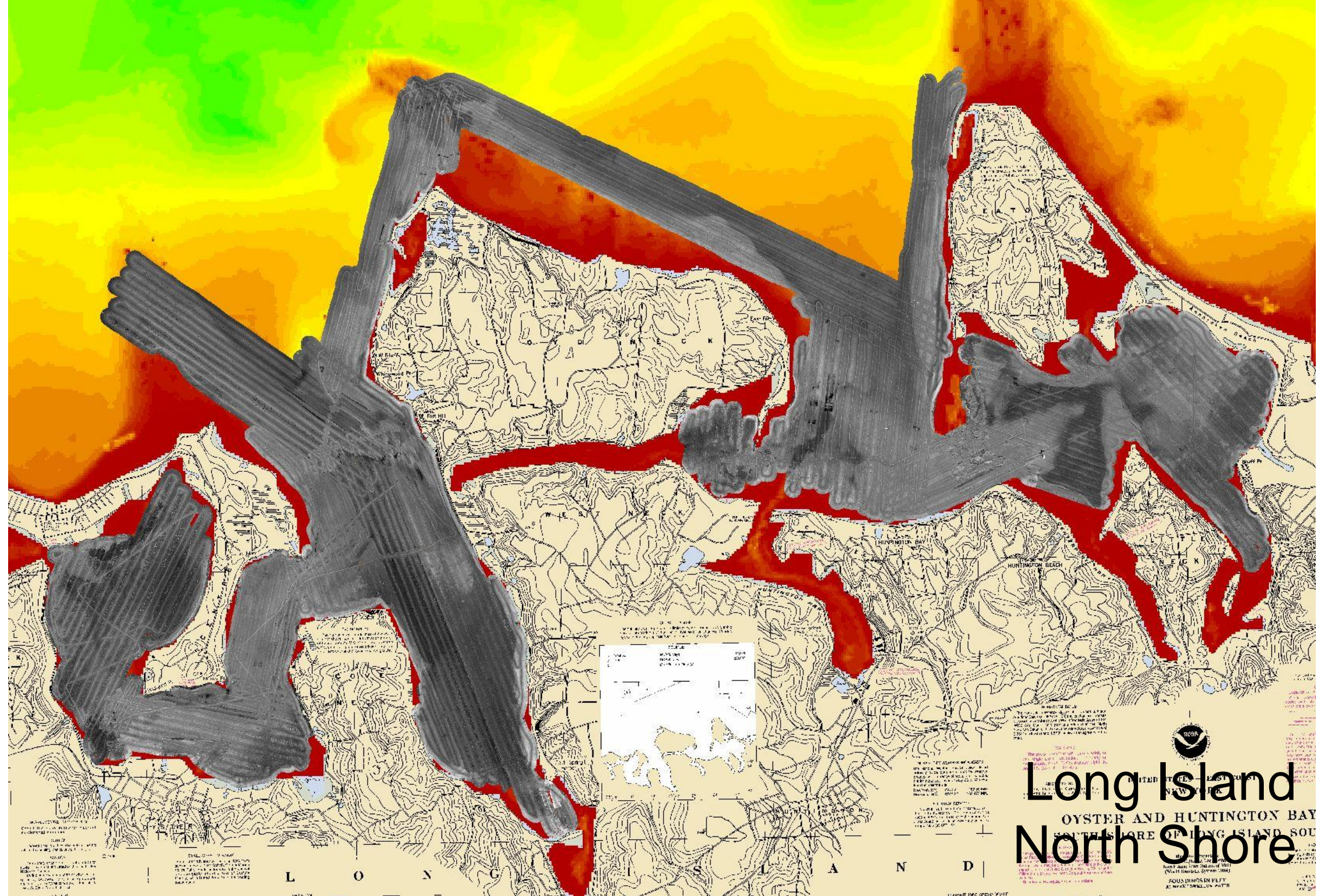




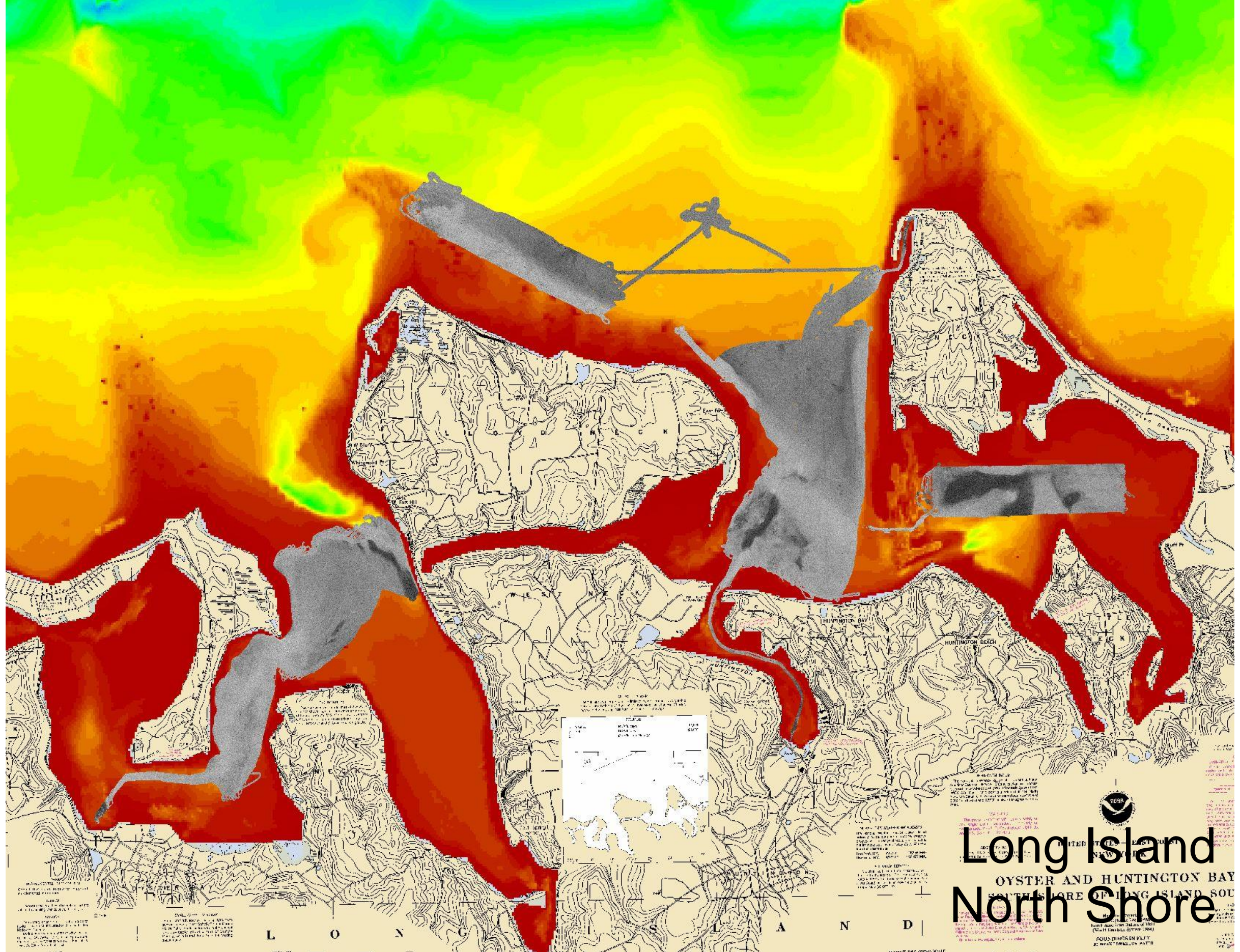




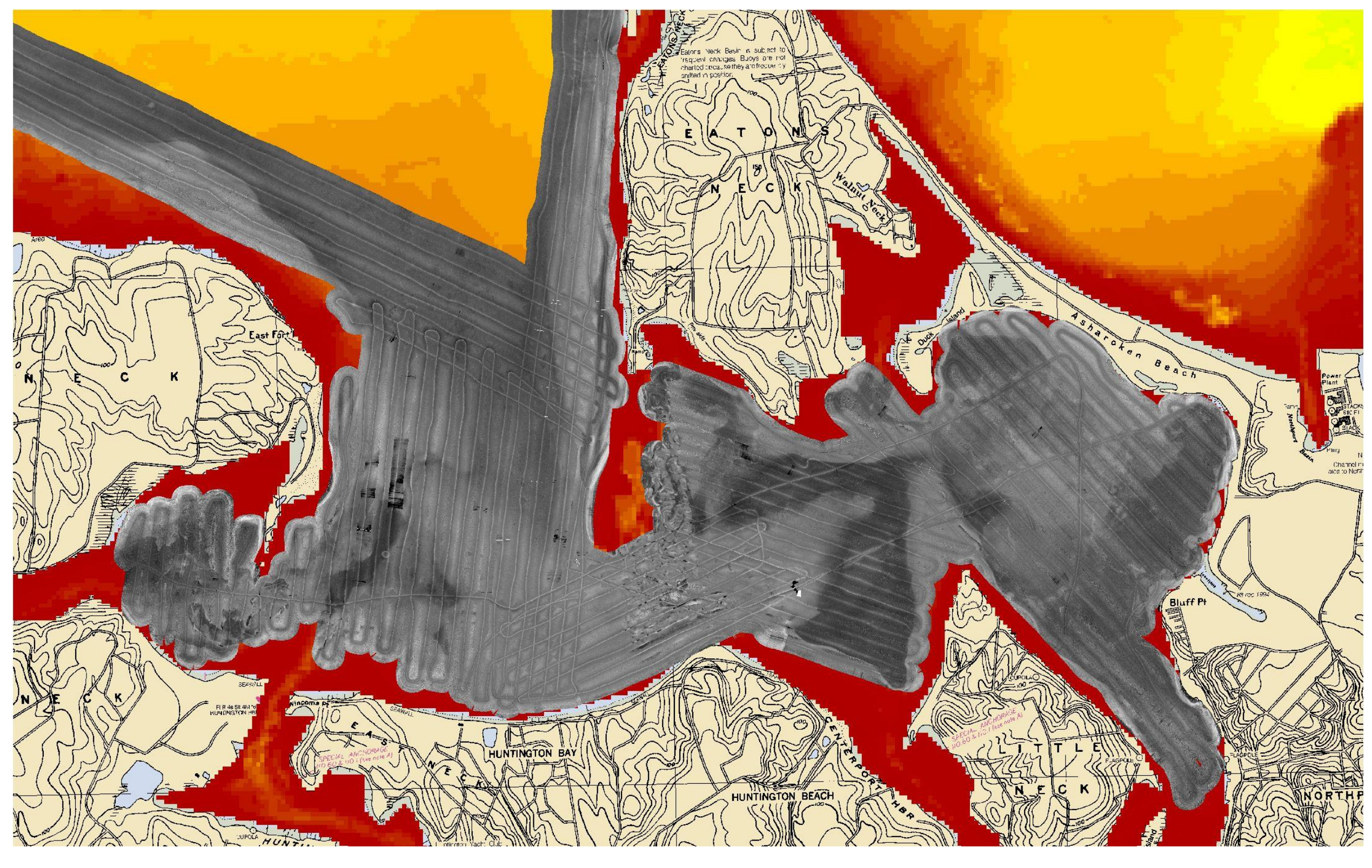

Huntington Bay 


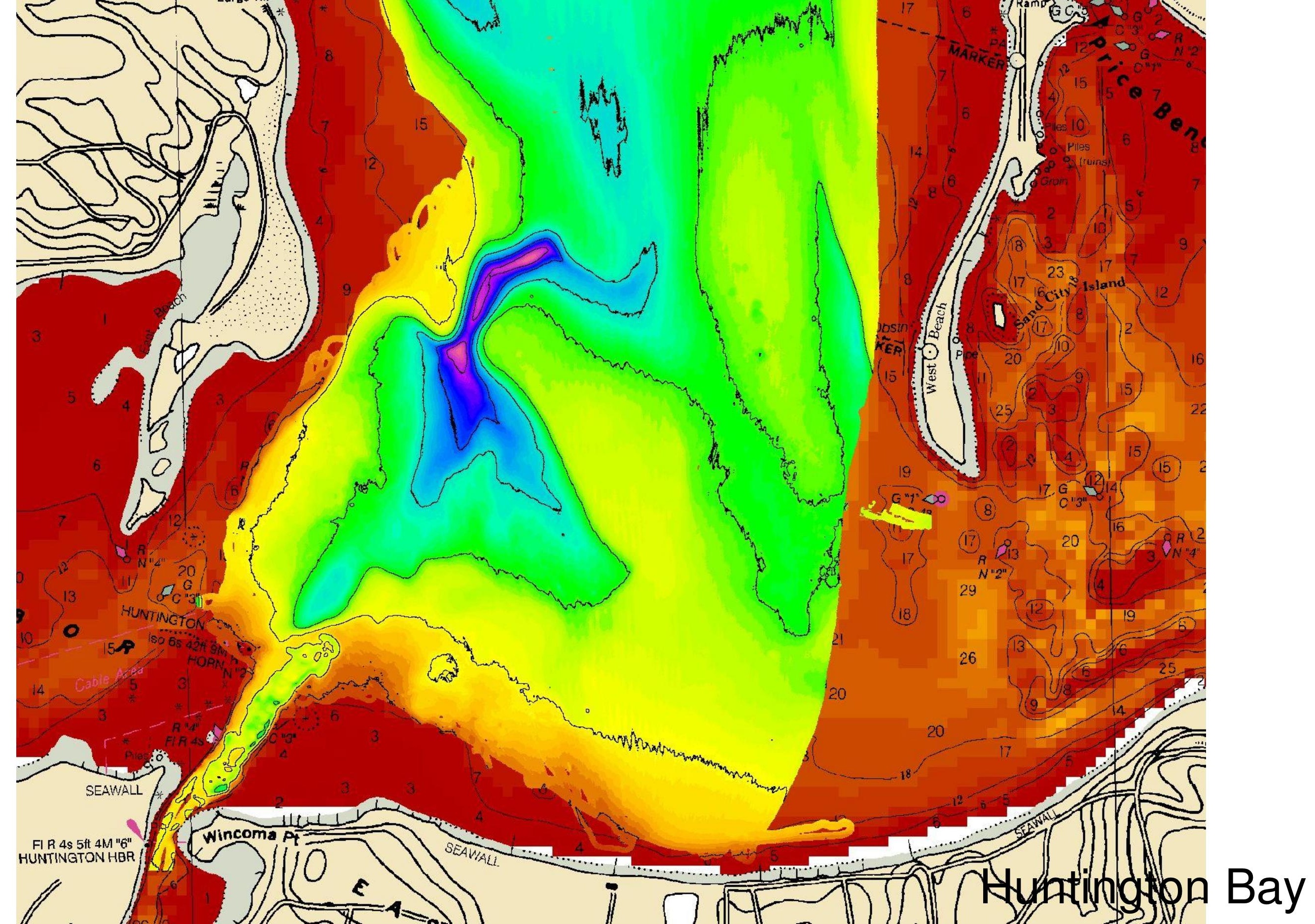




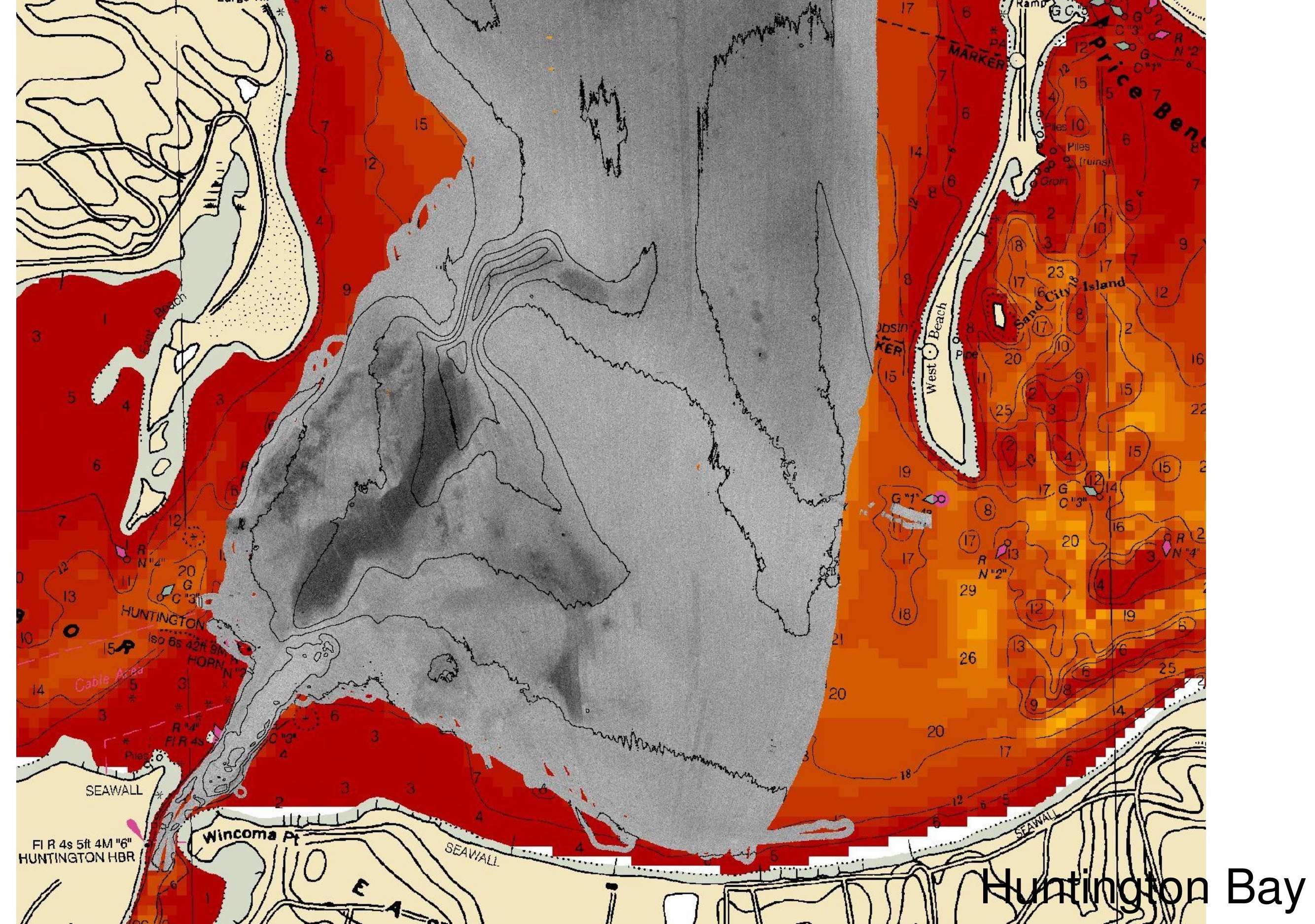




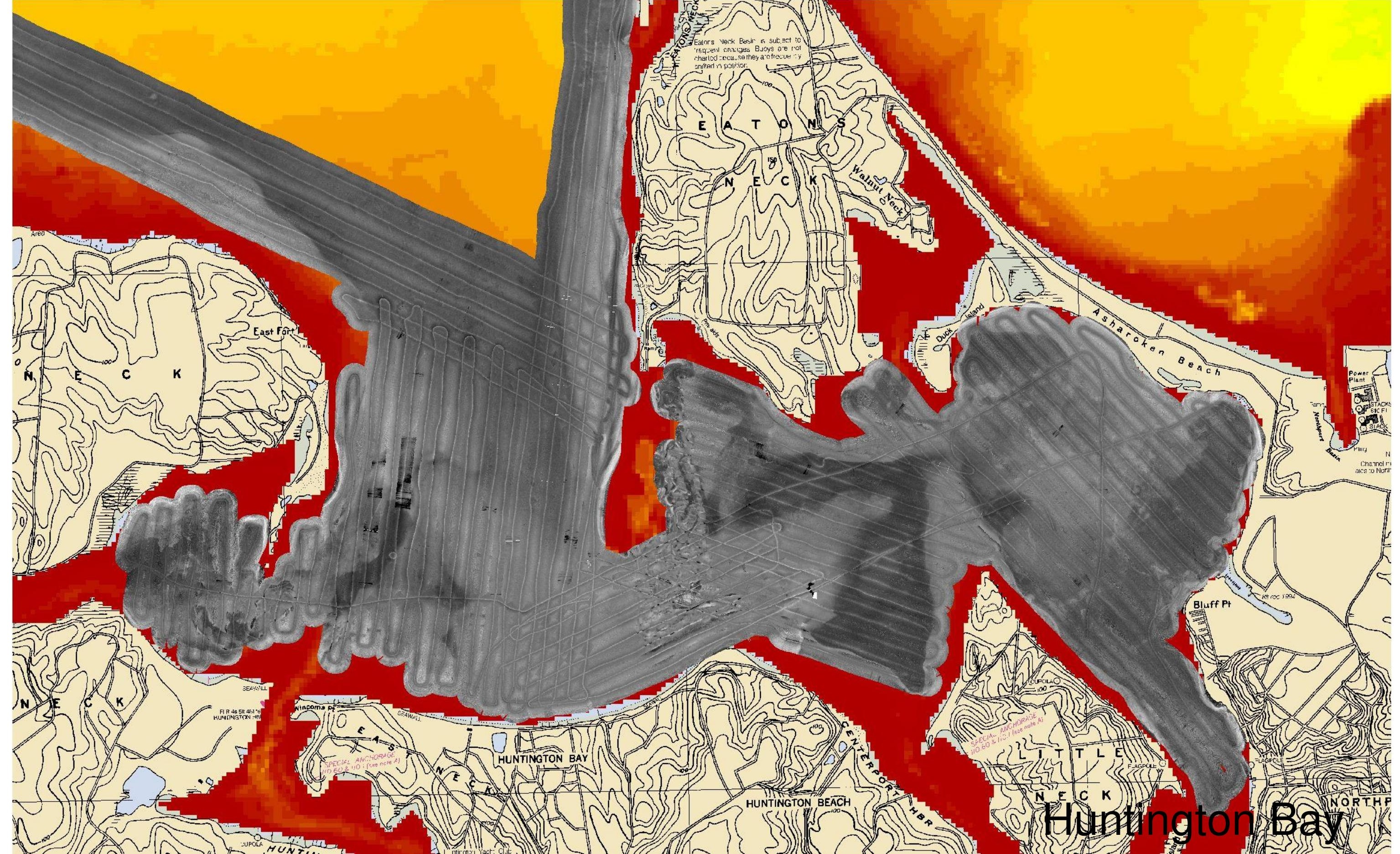




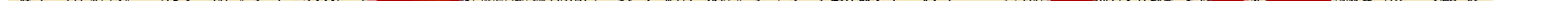


Results of statistical analysis of faunal species in grab samples.

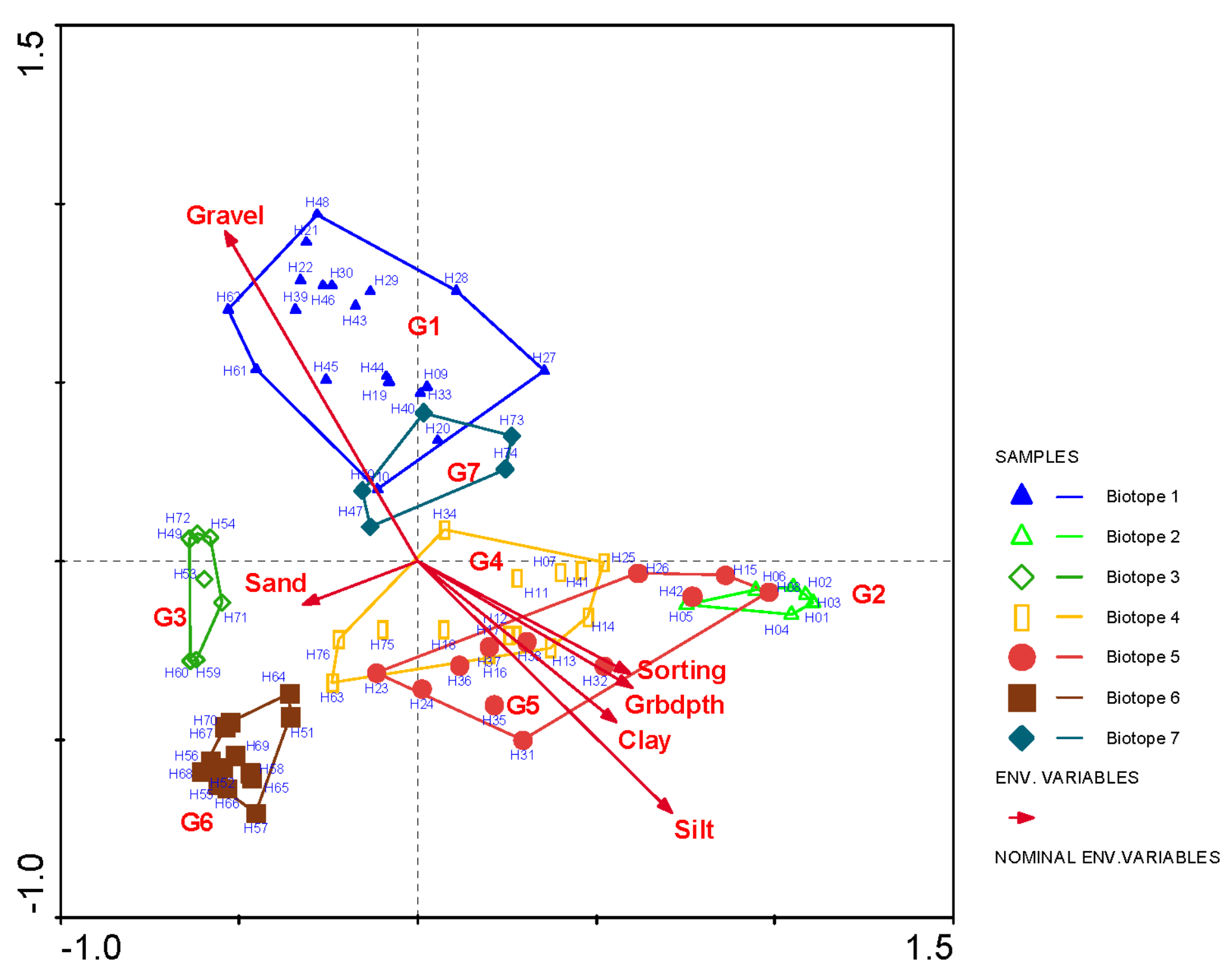

RDA analysis in Huntington Harbor. Sample labels are plotted next to points. Samples are colored by membership in the 7 biotope clusters. Sample proximity implies similarity.c 
Results of statistical analysis of faunal species in grab samples.

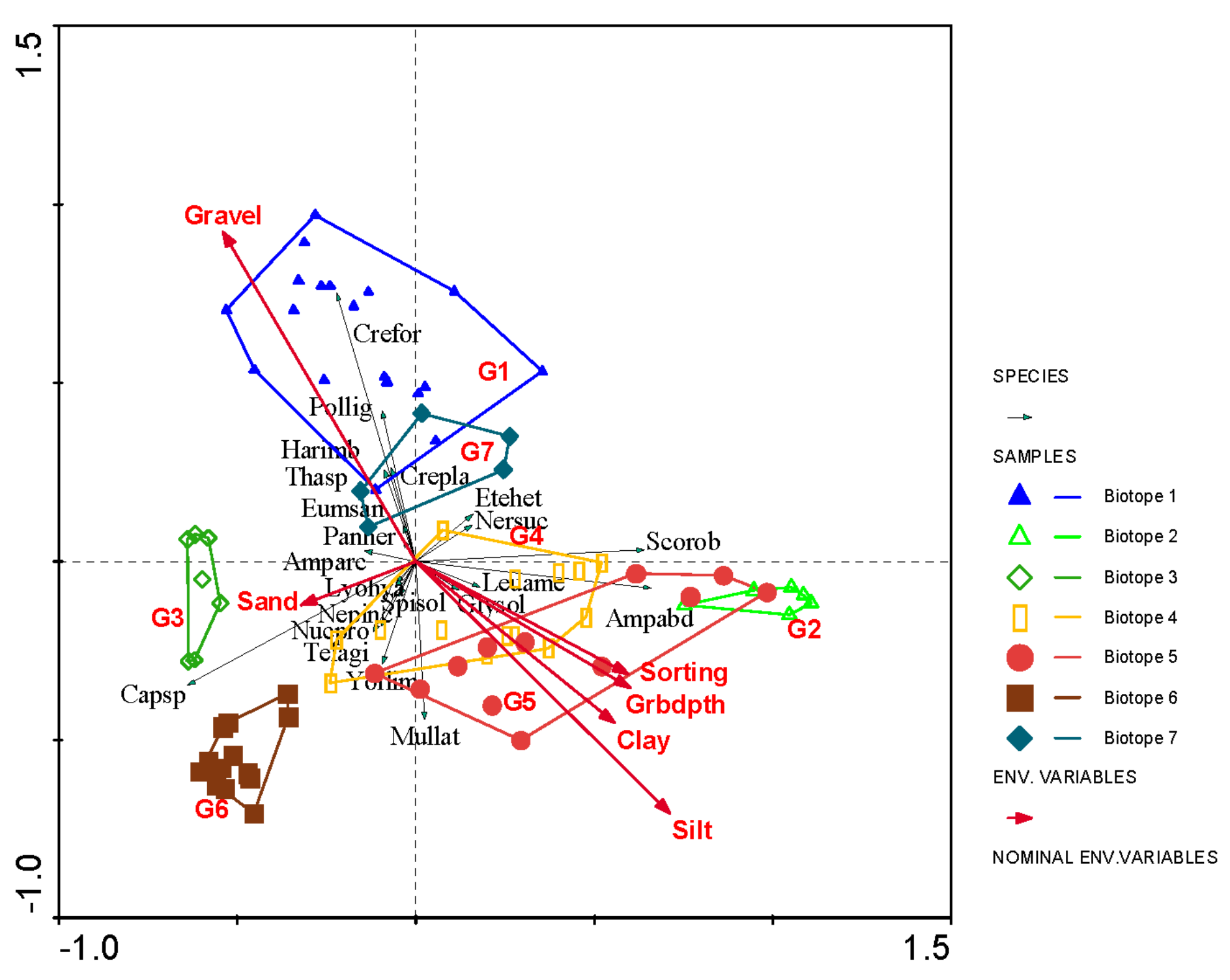

Black species arrows point in the direction of the steepest increase across the diagram. Angles between species arrows indicate correlations between the species. Sample proximity implies similarity. The 22 species listed are those for which $15 \%$ or greater of their variance is displayed in these first two dimensions. 
Species in two of the biotopes:
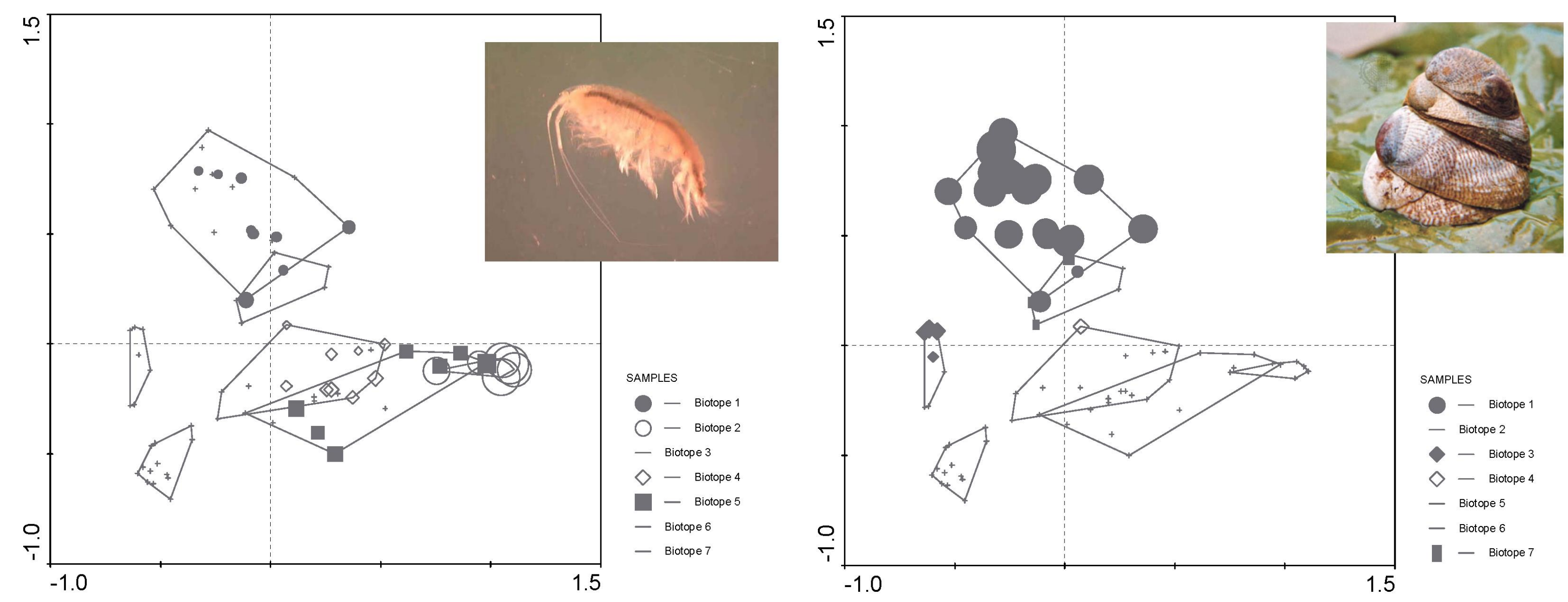

Relative abundance of the amphipod Ampelisca abdita (left) and the slipper shell Crepidula fornicate (right) in the Huntington Harbor biotopes. Points represent samples. Symbol diameters are proportional to relative abundance. 
Species in two more of the biotopes:
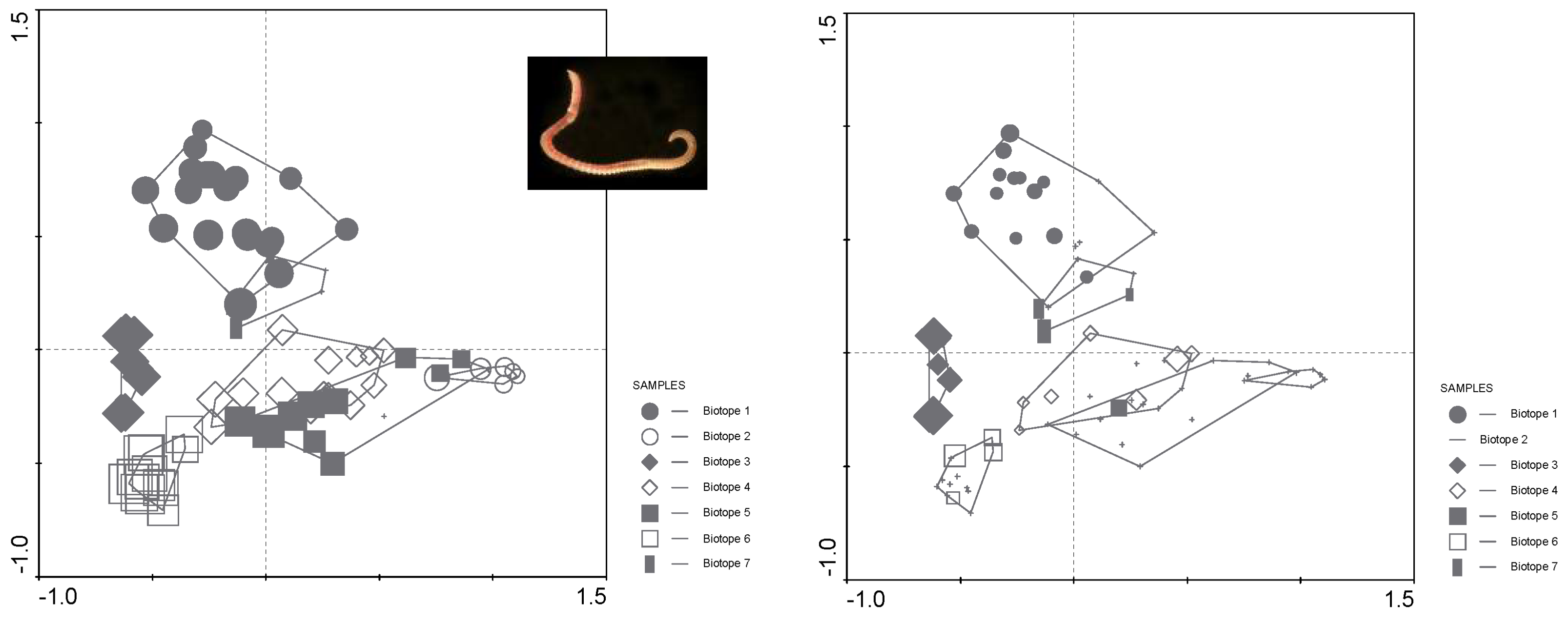

Relative abundance of the opportunistic polychaete Capitella sp (left) and the polychaete Ampharete arctica (right) in the Huntington Harbor biotopes. Points represent samples. Symbol diameters are proportional to relative abundance. 

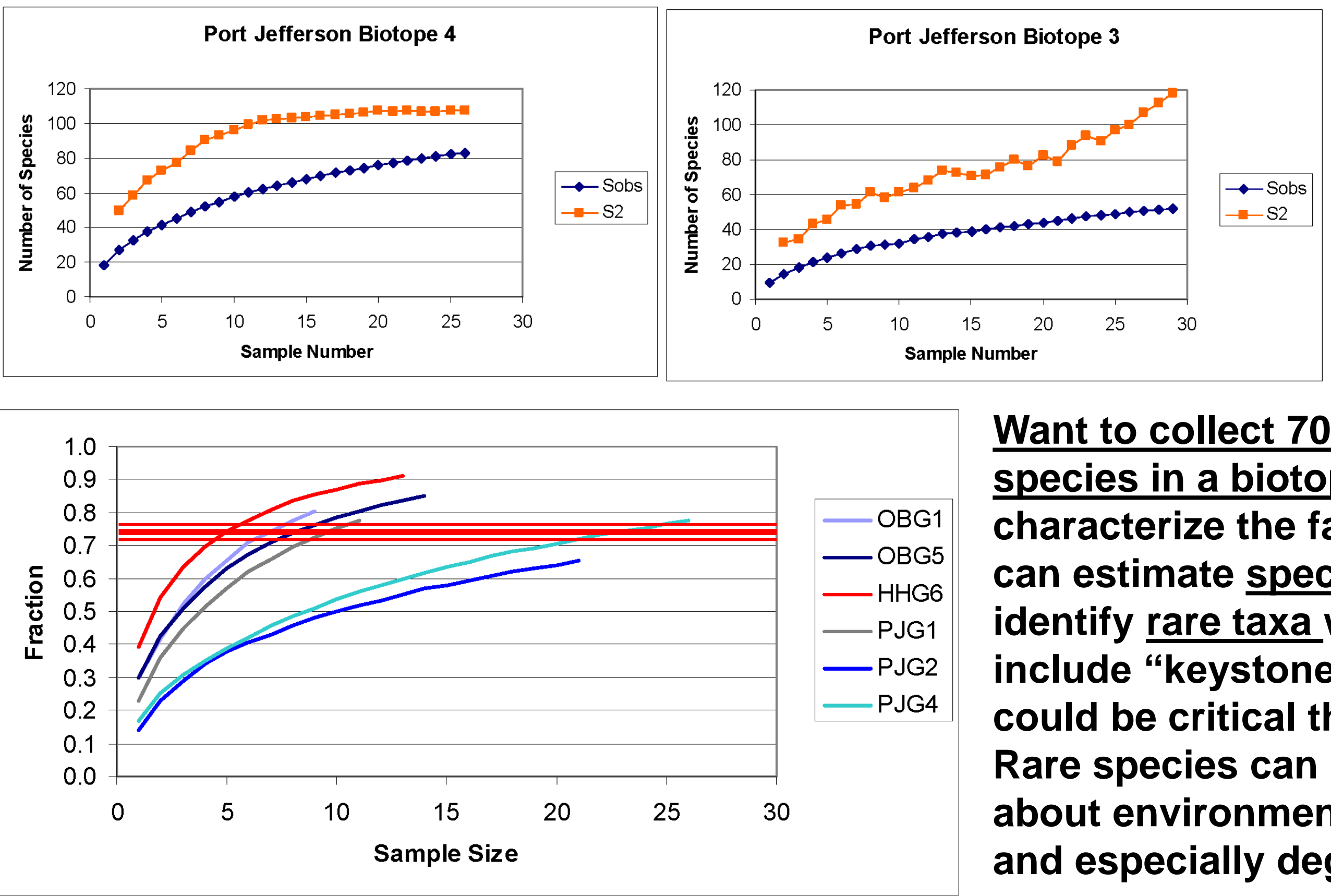

Want to collect $70-75 \%$ of the species in a biotope to adequately characterize the fauna, then we can estimate species richness and identify rare taxa which may include "keystone" species that could be critical the community. Rare species can also tell us about environmental conditions and especially degradation. 


\section{Long Island Sound}

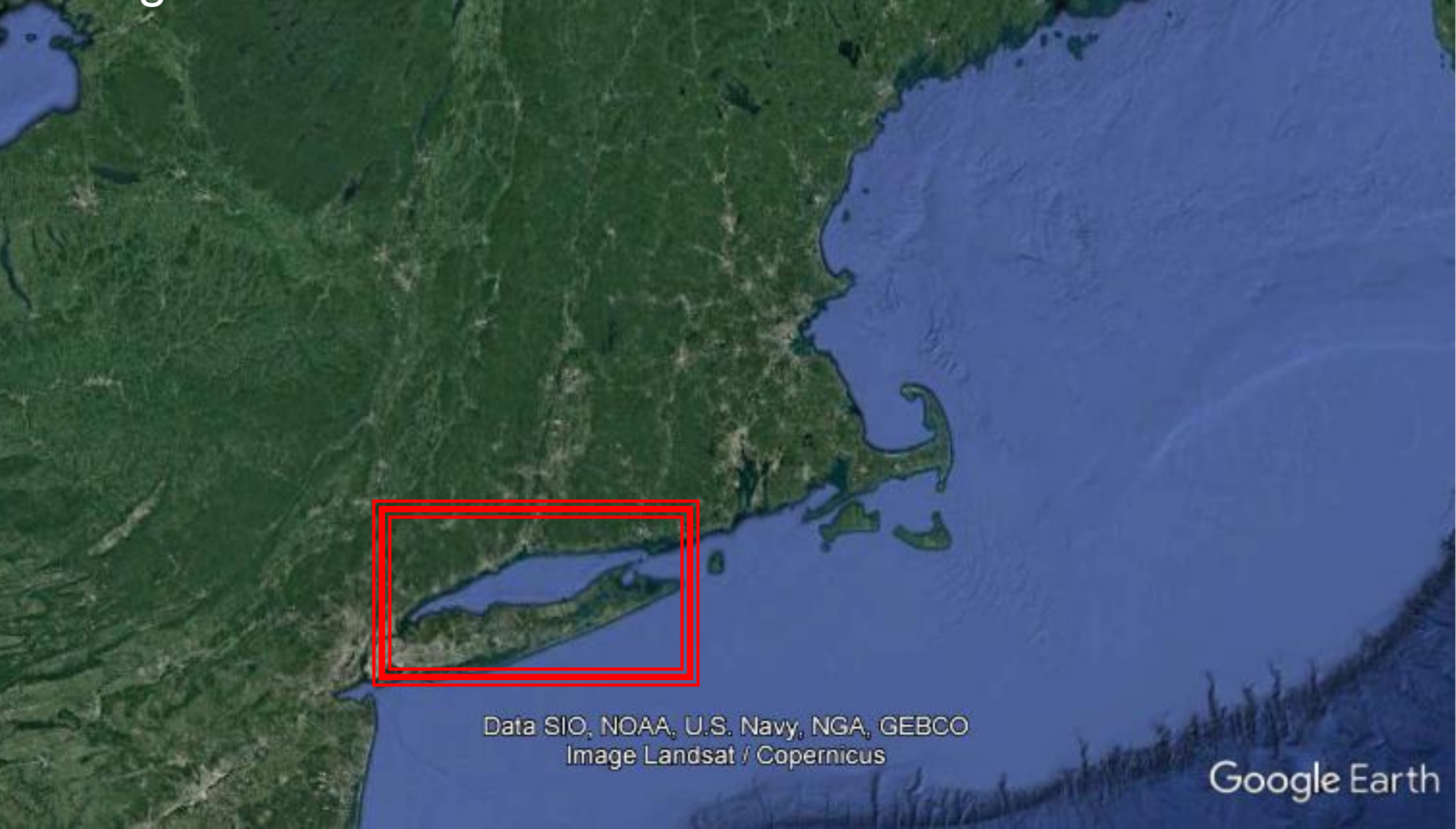


Long Island Sound, Pilot (Phase 1)

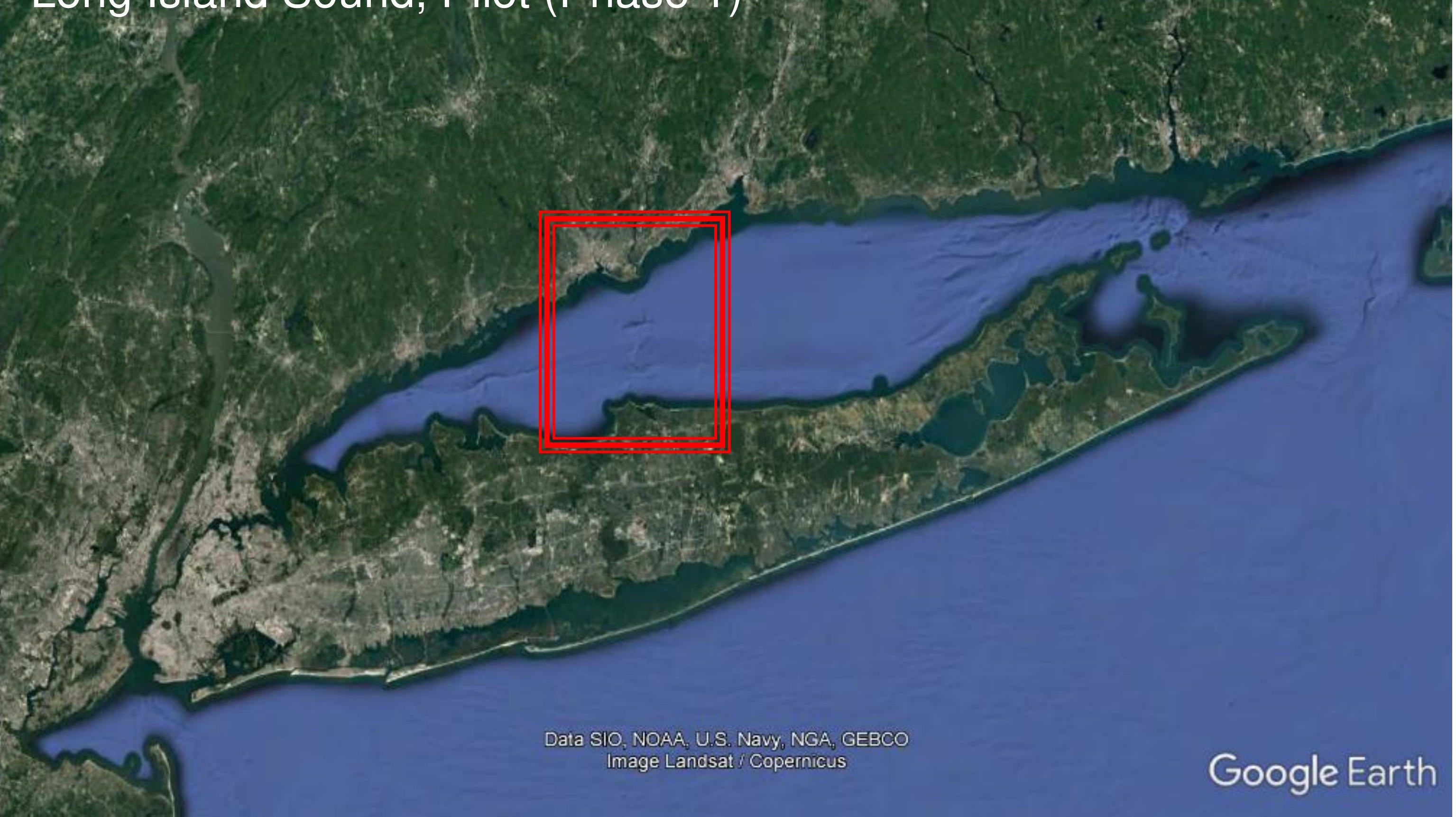




$$
15
$$




\section{Long Island Sound, Pilot (Phase 1) - SoMAS Benthic Samples}

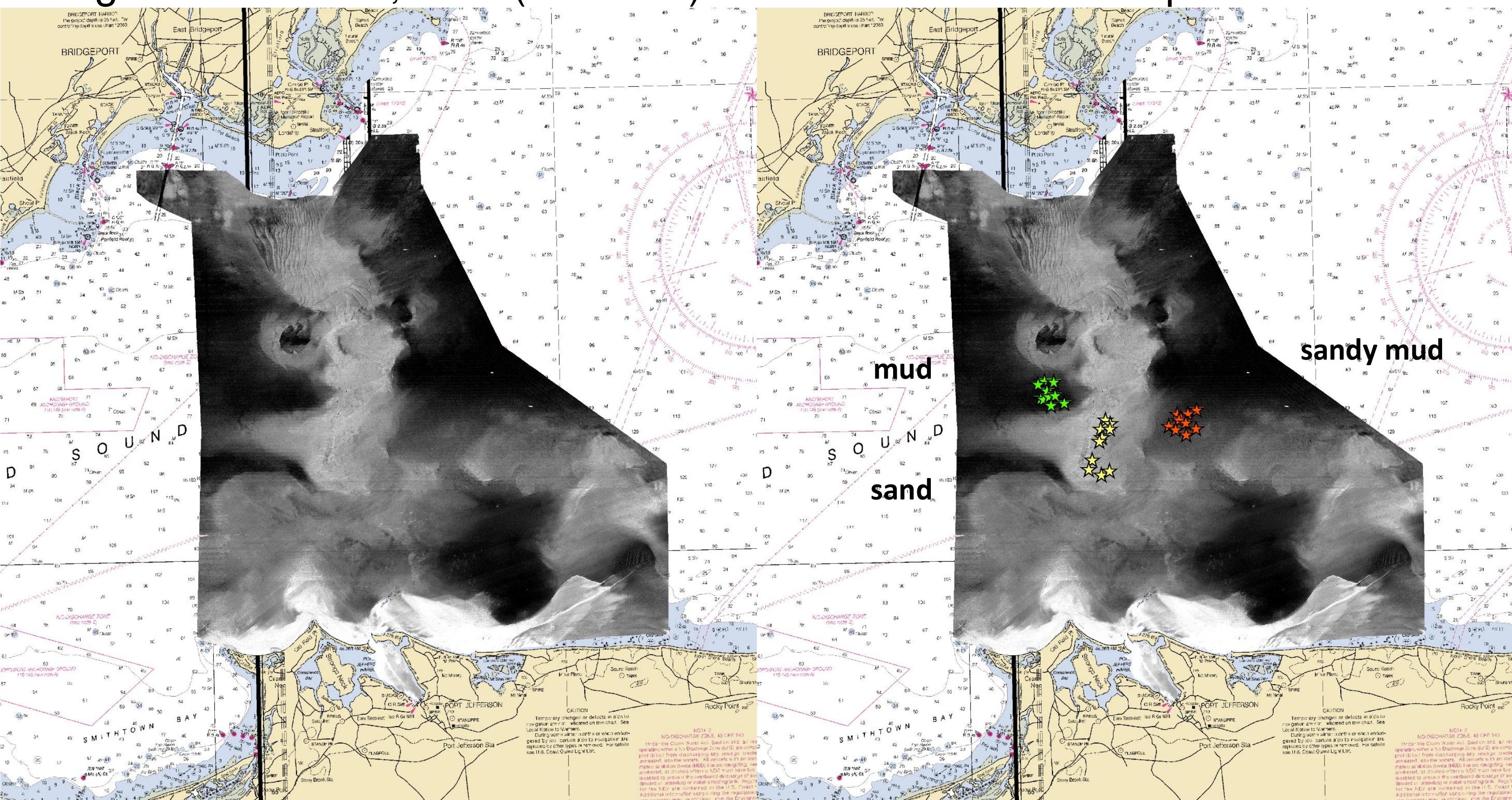




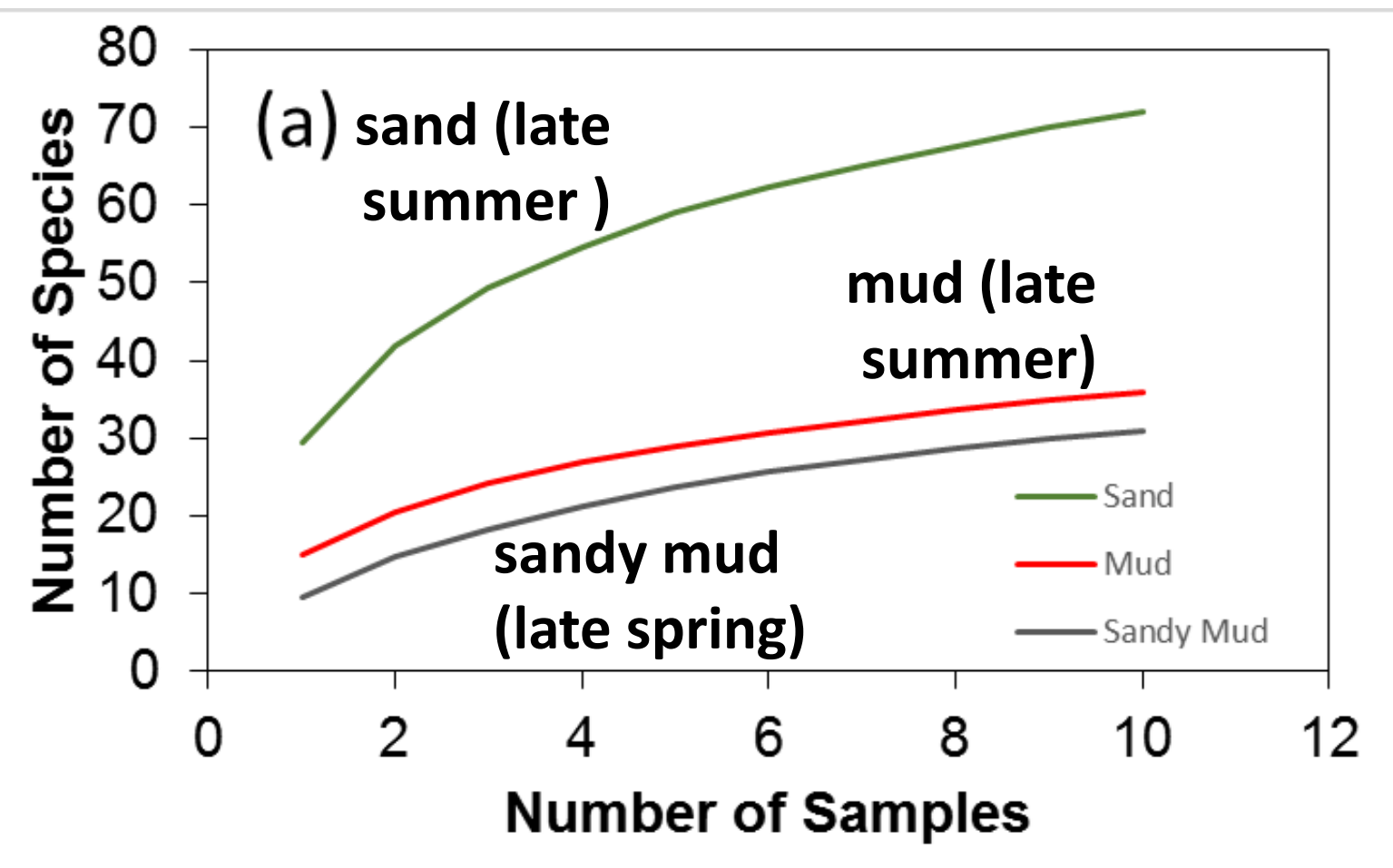

Three replicate samples in a habitat, a value often used in benthic studies, would recover only $13-50 \%$ of the species present. Even at 10 samples, there is evidence for under sampling in many of the habitats. In the present study, for example, 10 samples recovered only about $60 \%$ of the species in the Mud area.

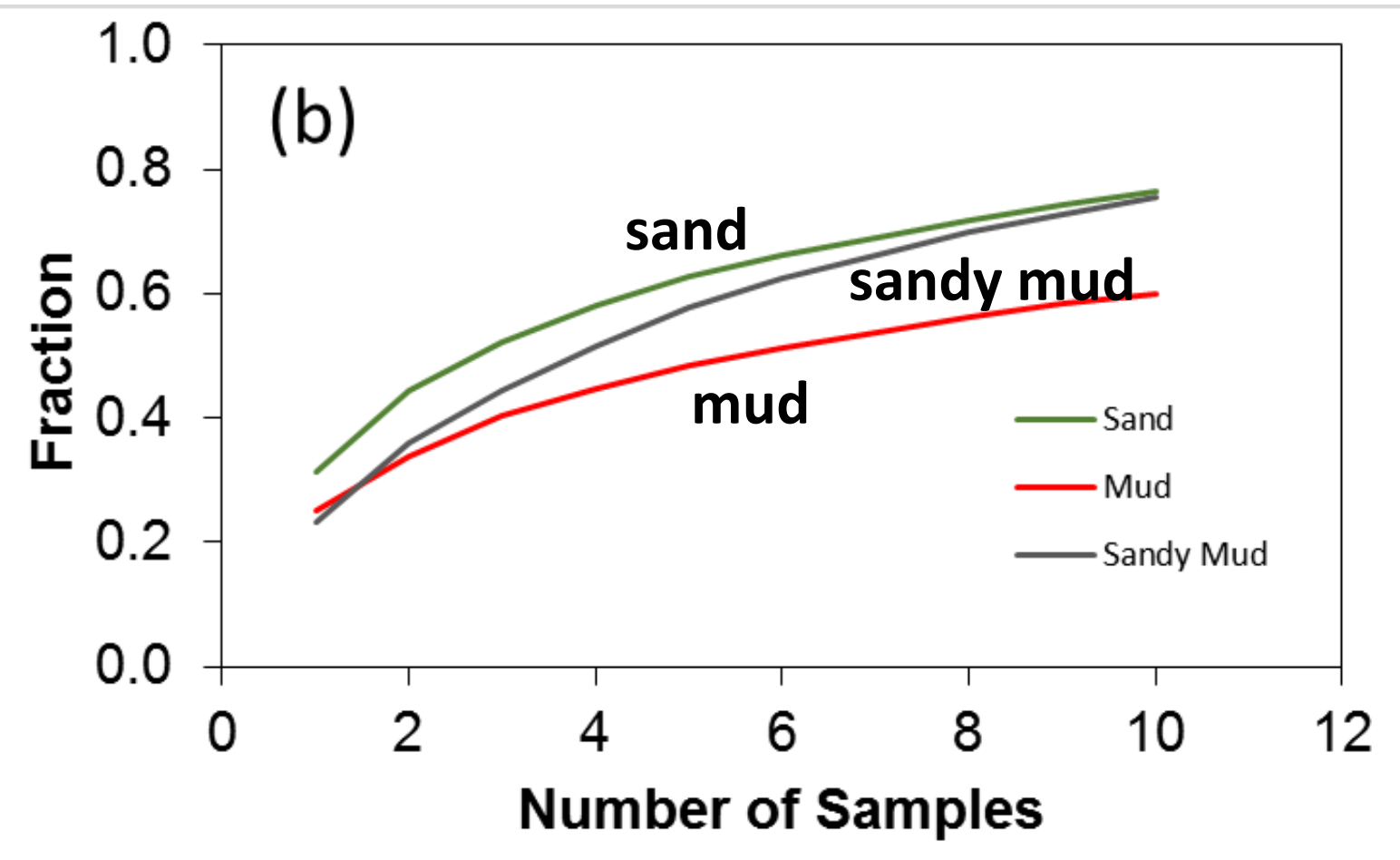

\begin{tabular}{l|c|c|c|c|} 
& Sand & Mud & $\begin{array}{c}\text { Sandy } \\
\text { Mud }\end{array}$ & Total \\
\hline $\begin{array}{l}\text { Total } \\
\text { Species }\end{array}$ & 72 & 36 & 31 & 95 \\
\hline Rare $(<1 \%)$ & 60 & 22 & 17 & 81 \\
\hline $\begin{array}{l}\text { Common } \\
(>1 \%)\end{array}$ & 12 & 14 & 14 & 14 \\
\hline
\end{tabular}




\section{Why Collect Rare Species?}

- Rare taxa may include functionally important, "keystone" species, critical to energy and material flows (Hooper et al. 2005)

- Rare species are often more sensitive to disturbances than abundant species. Therefore, occurrence of rare species can serve as reliable indicators of environmental health (Cao et al. 1998; Gaston 1998)

- Unfortunately, rare species are largely ignored in assessment and monitoring programs; sacrificed for cost-efficiency (Cao et al. 1998) 
Long Island Sound, Phase 2

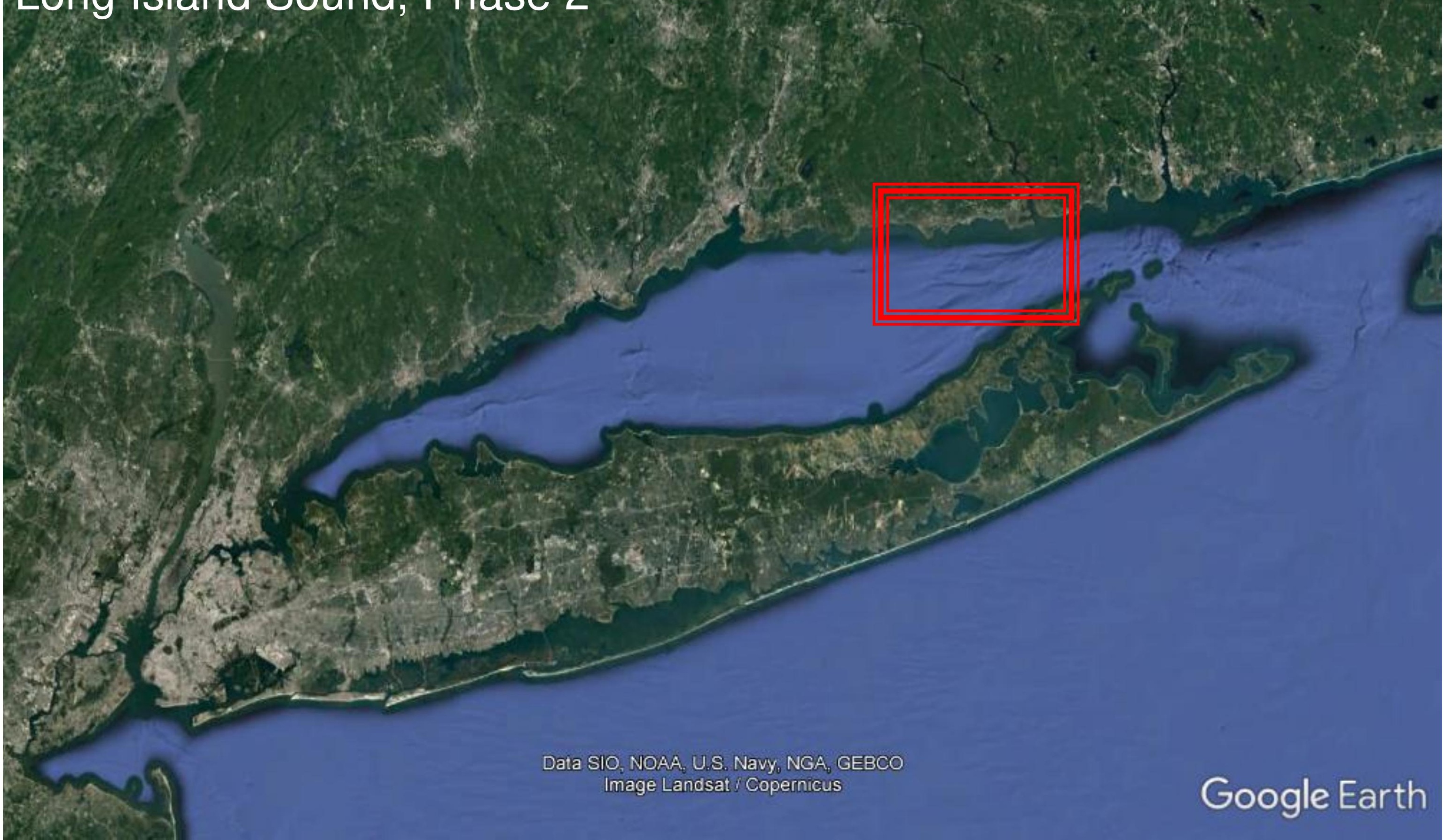




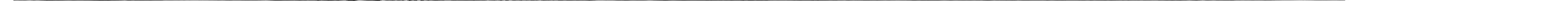


Banchan Dunes nove $40-100$

\section{1}

In to the SIN in the $\sim 10$ years

between surveys due to

repeated tidal flows!

(1) 


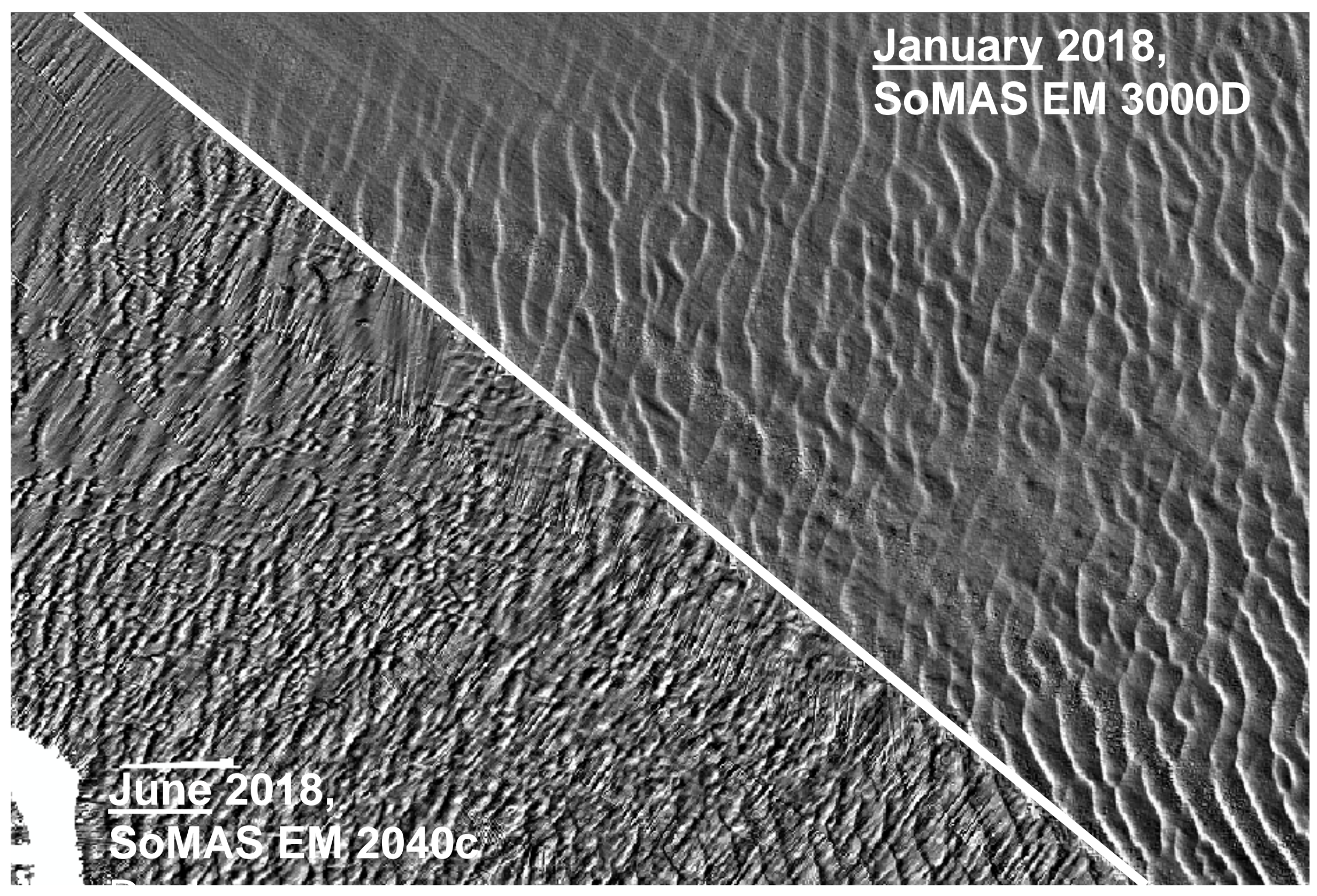


USGS 01194750 CONNECTICUT RIYER AT ESSEX, CT
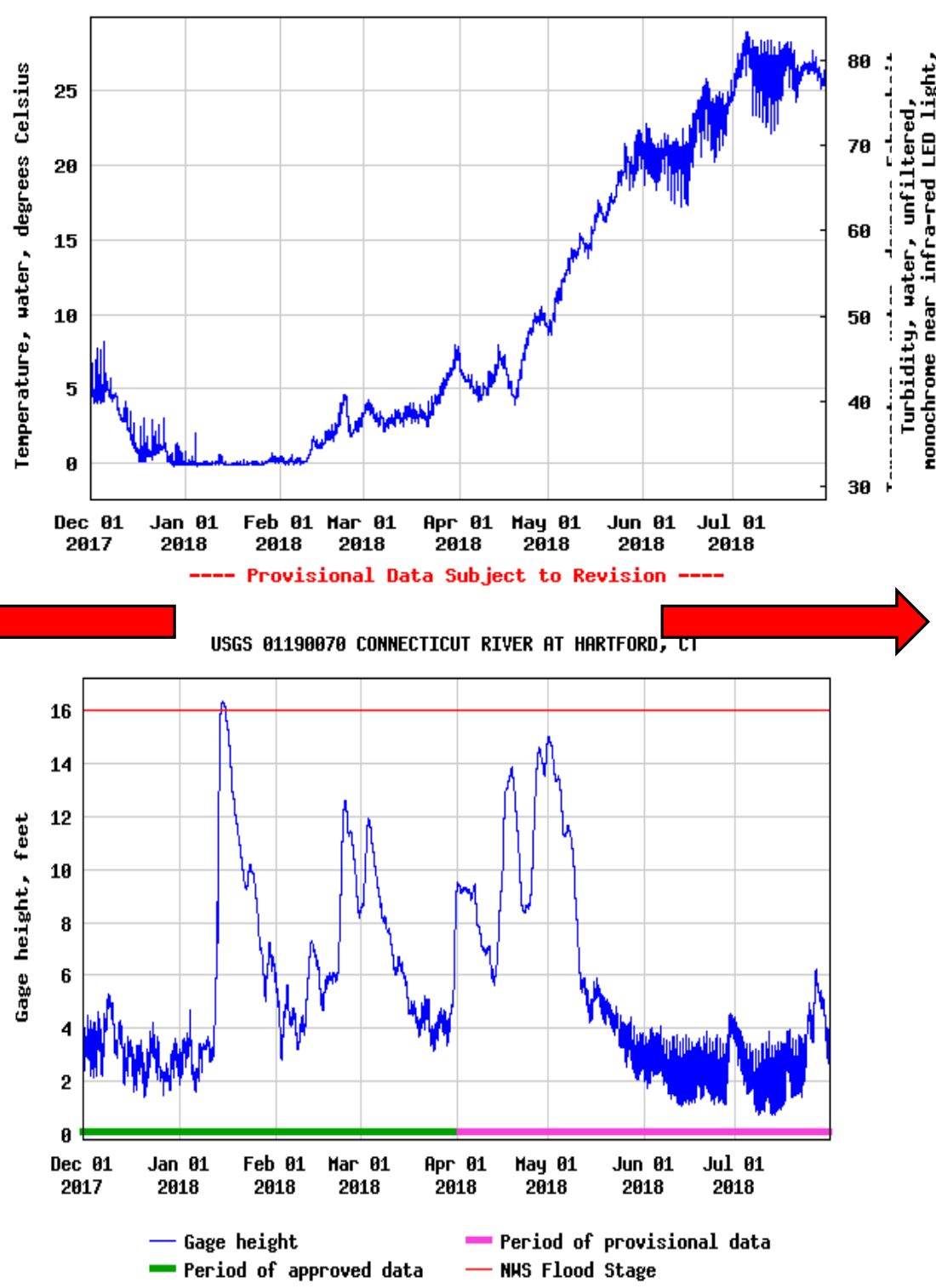

USGS 01193850 CONMECTICUT RIVER AT MIDDLE HADDAM, CT

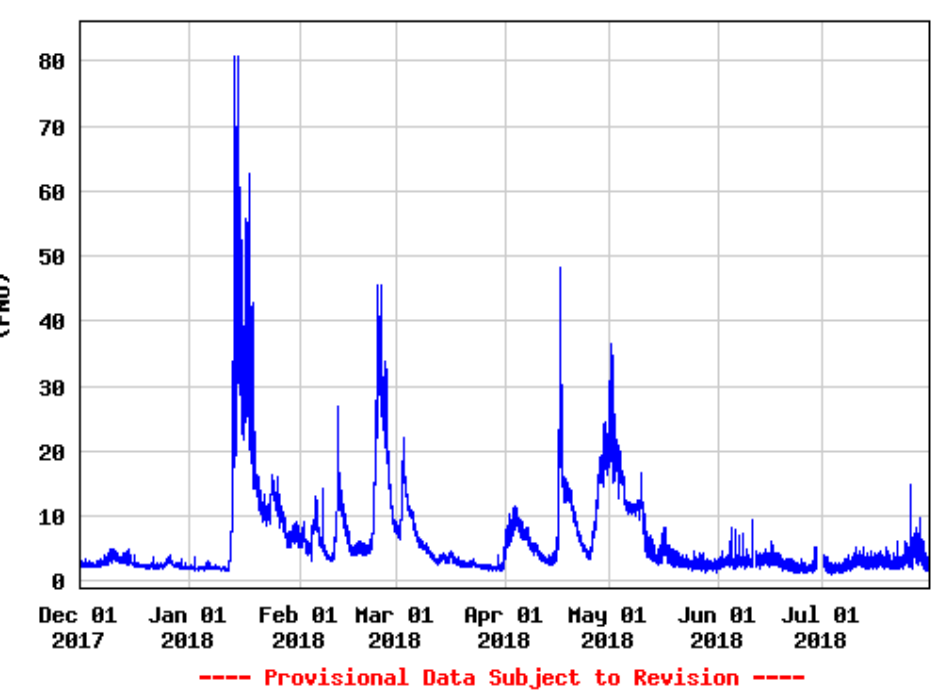

USGS 0118480 CONMECTICUT RIYER AT THOHPSONYILLE, CT

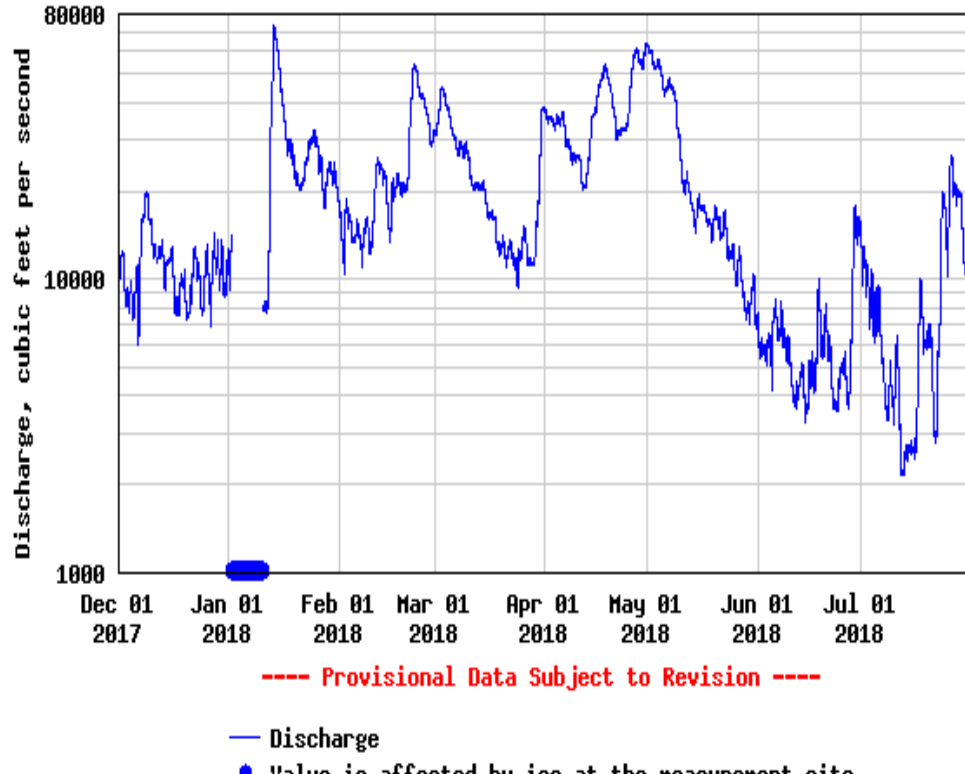




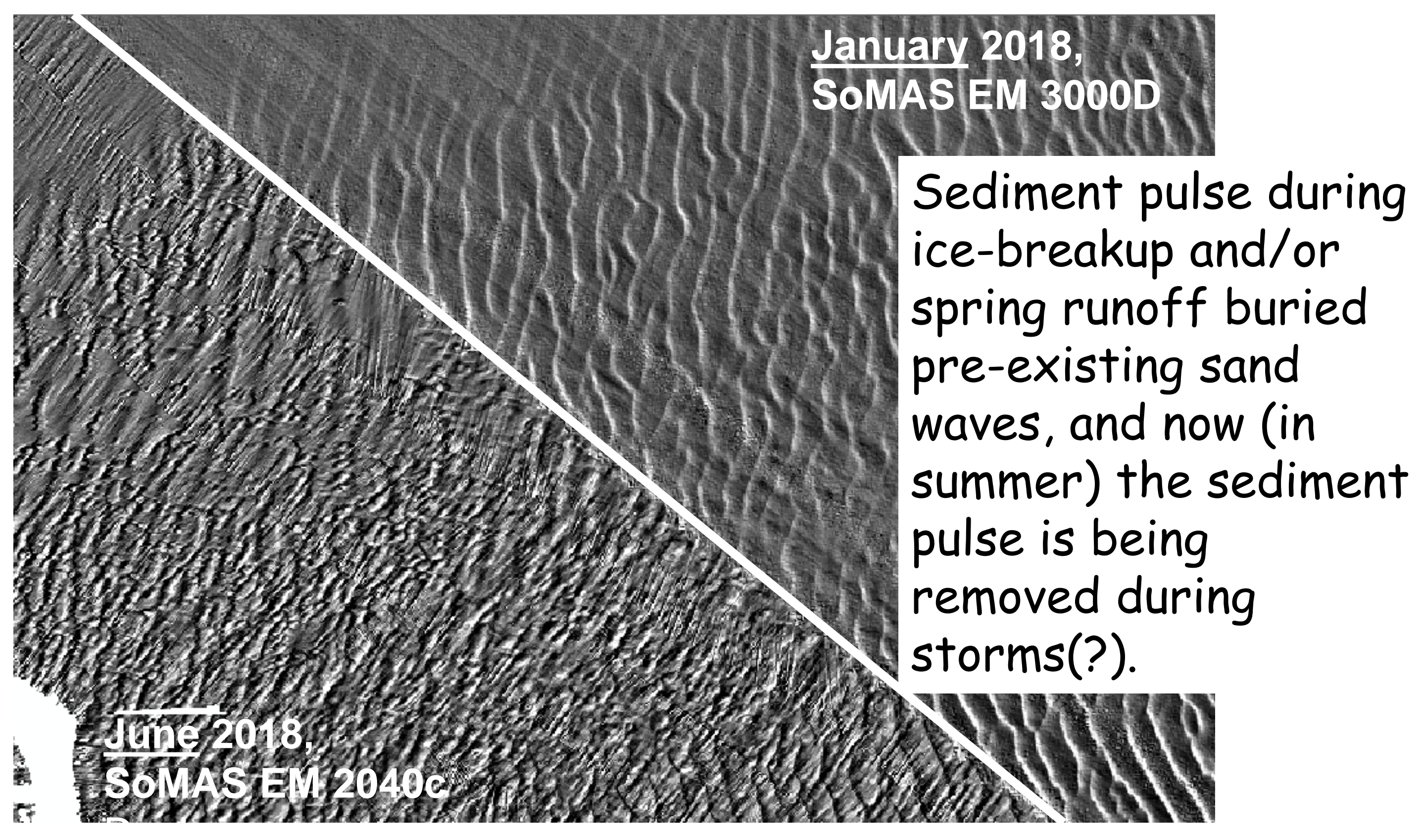




\section{Conclusions:}

- The distribution of acoustic backscatter patterns contains information about the distribution of benthic organisms and should guide sampling efforts that characterize benthic habitats.

- Backscatter distribution patterns help to explain a large percentage of the benthic faunal and do better at predicting faunal distribution patterns than do feature-based schemes.

- Serendipitous repeat surveys help to demonstrate the nature of sediment-transport events. 